\title{
Oxidative Stress and NO Generation in Cerulein-Induced Rat Pancreatitis
}

\section{Katharina Tiemann ${ }^{1}$, Jürgen Schnekenburger ${ }^{2}$, Verena Schick ${ }^{3}$, Uta Demus ${ }^{4}$, Ursula Müller-Werdan ${ }^{5}$, Dmitri Atiakshin ${ }^{6}$, Werner Boecker ${ }^{1}$, Vera Samoilova ${ }^{1}$ and Igor Buchwalow*1}

${ }^{1}$ Institute for Hematopathology, Germany

${ }^{2}$ Biomedizinisches Technologiezentrum der Medizinischen Fakultät, Germany

${ }^{3}$ Department of Medicine B, University Hospital Muenster, Germany

${ }^{4}$ Gesellschaft zurFörderungvonMedizin, Bio- und Umwelttechnologien, Germany

${ }^{5}$ Chair of Geriatrics, Charité-Universitätsmedizin Berlin, and Protestant Geriatric Centre, Germany

${ }^{6}$ Research Institute of Experimental Biology and Medicine, Burdenko Voronezh State Medical University, Russia

Received: May 01, 2018; Published: May 11, 2018

*Corresponding author: Igor Buchwalow, Institute for Hematopathology, 22547 Hamburg, Germany

\begin{abstract}
The interaction between nitric oxide (NO) and superoxides is critical in the development of pancreatitis. Previously, we reported on the up-regulation of oxidative stress and NO-synthase (NOS) in the human chronic pancreatitis and in an animal model of pancreatitis induced by pancreatic duct ligation (PDL) in rats. We have shown that oxidative stress runs ahead of NOS up-regulation, which implies that the NO enhancement in the course of pancreatitis is likely to be an adaptive mechanism aimed at maintaining the homeostatic cellular level of the bioactive NO. Here, we report on the expression of NOS and oxidative stress markers (nitrotyrosine and 8-hydroxyguanosine) in the course of cerulein-induced acute pancreatitis in rats. We found that the pattern of superoxide's/NO interaction in this model of acute pancreatitis is similar to that in the PDL-induced rat pancreatitis and in the human chronic pancreatitis. It means that cerulein-induced acute pancreatitis like the PDL-induced pancreatitis is a proper model for further studies of pancreatitis development in humans.
\end{abstract}

Keywords: Acute Pancreatitis; Nitric Oxide Synthase; Oxidative Stress

\section{Introduction}

Acute pancreatitis is a sudden inflammation of the pancreas. There has been increasing awareness over the last 20 years of the role played by oxidative stress in acute pancreatitis. Oxidative stress can produce a higher level of superoxides, such as multiple types of reactive oxygen species (ROS) [1]. The interaction between superoxides and nitric oxide (NO) is critical in the development of pancreatitis [2] and, as a result, the cell is getting short of bioactive NO and responses with up-regulation of NOS [3]. We have shown earlier that oxidative stress runs ahead of NO-synthase (NOS) upregulation in pancreatic inflammation [4,5], which implies that the increased NO generation in the course of acute pancreatitis is likely to be an adaptive mechanism aimed at maintaining the homeostatic cellular level of the bioactive NO. Whereas deleterious effects of superoxides in acute pancreatitis are firmly established, data from experimental models of acute pancreatitis are limited and still remain ambiguous and species specific [6].

The obvious controversies and confusions in this area necessitated a re-examination of the current knowledge about NOS localization in pancreas employing state-of-the-art immunohistochemical technology employing highly sensitive method of tyramine signal amplification (TSA). This platform was used in this study on cerulean-induced pancreatitis in rats to characterize dynamical changes in the expression of NOS and oxidative stress markers-nitro tyrosine and 8-hydroxyguanosine (80HG). The pattern of superoxides/NO interaction in the model of cerulein-induced acute pancreatitis in rats was found to be similar to that reported earlier by us for the rat model of pancreatitis induced by pancreatic duct ligation (PDL) as well as for the human chronic pancreatitis, which implies the applicability of this model for further studies of pancreatitis development in humans.

\section{Materials and Methods}

\section{Animals}

The investigation conforms with Guide for the Care and Use of Laboratory Animals published by the US National Institutes of Health (NIH Publication No. 85-23, revised 1996) and was per- 
formed in accordance with the guidelines of the Institutional Animal Care Committee, University of Muenster. The animal experiments were performed under the permission A25/2005 from the state NRW authority.

\section{Supramaximal Secretagogue Stimulation in vivo}

Male Wistar rats weighing 280-300g and kept in an environment of constant temperature, humidity and day-night cycle was used. Rats were anaesthetized with pentobarbital $30 \mathrm{mg} / \mathrm{kg}$. A cannula was placed into the jugular vein, and the animals were infused with supramaximal concentrations of cerulein $(10 \mu \mathrm{g} / \mathrm{kg} / \mathrm{h})$ for up to $48 \mathrm{~h}$. Saline infused animals served as controls $[7,8]$. After exsanguination under ether anesthesia the pancreas was rapidly removed, trimmed of fat, fixed in buffered $4 \%$ formaldehyde and routinely embedded in paraffin. The part of the pancreas probes was frozen in liquid nitrogen and stored at $80^{\circ} \mathrm{C}$ for later protein analysis [9]. Paraffin tissue sections ( $4 \mathrm{~m} \mu$ thick) mounted on Polysine microslides (Menzel Gläser, Braunschweig, Germany) were deparaffinized with xylene and graded ethanol, while antigen retrieval was achieved by heating the sections in $10 \mathrm{mM}$ sodium citrate buffer, $\mathrm{pH} 6.0$, at $95^{\circ} \mathrm{C}$ for $30 \mathrm{~min}$ in a domestic vegetable steamer.

Subsequently, sections were encircled with a water-repellent PAP-pen (Dianova, Hamburg, Germany) and rinsed with PBS. PBS was used for all dilutions and washing steps. Immunostaining was performed according to the standard protocol routinely used for immunohistopathology [10]. We recently reported that endogenous Fc receptors in routinely fixed cells and tissue probes do not retain their ability to bind Fc fragments of antibodies [11]; therefore, blocking the endogenous Fc receptors prior to incubation with primary antibodies was omitted.

\section{Antibodies and Immunohistochemical Staining}

After antigen retrieval, sections were immunoreacted with primary antibodies over night at $4^{\circ} \mathrm{C}$. Characterization of rabbit primary polyclonal antibodies recognizing $\mathrm{NOS}_{1}, \mathrm{NOS}_{2}$ and $\mathrm{NOS}_{3}$ (Transduction Laboratories, Lexington, KY, USA) including Western blotting was described elsewhere. Primary anti-NOS antibodies were diluted to a final concentration of $0.2-0.5 \mu \mathrm{g} / \mathrm{ml}$. Goat antiserum to 8-hydroxyguanosine (80HG, a marker of oxidative damage to nucleic acids, purchased from Serotec Ltd, Oxford, UK) was applied at a dilution of 1:200. Rabbit antiserum to nitro tyrosine (Molecular Probes, Leiden, The Netherlands) was used at a dilution of 1:200. After immunoreactions with primary antibodies and following washing in PBS, the sections were treated for 10 min with methanol containing $0.6 \% \mathrm{H}_{2} \mathrm{O}_{2}$ to quench endogenous peroxidase.

For bright-field microscopy, bound rabbit primary antibodies were visualized with blast amplification Buchwalow and Boecker [10] employing correspondingly rabbit or mouse AmpliStain ${ }^{\mathrm{TM}}$ HRP conjugates (SDT GmbH, Baesweiler, Germany) Buchwalow et al. [5] and biotin-labeled tyramine in combination with HRP-avidin-biotin complex (Vectastain "Elite" ABC-kit, Vector Laboratories, USA). The HRP label was amplified with biotin-conjugated tyramine. Biotin-tyramine conjugate was synthesized from tyramine- $\mathrm{HCl}$
(Sigma, T2879) and biotin-succinimidyl ester (BIO-NHS, Pierce, Rockford, IL, 12062-22-0) in DMF Buchwalow and Boecker [10]. Incubation with biotin-tyramine conjugate was carried out at a dilution of 1:1000 in PBS in the presence of $0.02 \% \mathrm{H} 202$ for 3-8min. Consequently, biotin label was detected using HRP-avidin-biotin complex (Vectastain "Elite" ABC-kit, Vector Laboratories, USA).

To visualize the amplified HRP label, the samples were incubated with DAB or Nova Red Kits (both from Vector Laboratories) for 5-10min and counterstained with Ehrlich hematoxylin for 30sec. Tyramide amplification (TSA) allows an increase in the detection of an antigenic site up to 100-fold compared with the conventional indirect method with no loss in resolution. For fluorescent visualization of bound goat primary antibodies, bound goat anti80HG antibodies were visualized with FITC-conjugated donkeyanti-goat antibodies (Dianova, Hamburg, Germany). Finally, samples were counterstained for $15 \mathrm{sec}$ with DAPI $(5 \mu \mathrm{g} / \mathrm{ml}$ PBS; Sigma) and mounted with Vecta Shield (Vector Laboratories, USA). The controls were omission of incubation with primary antibodies or substitution of primary antibodies with the corresponding IgG at the same final concentration as the primary antibodies.

\section{Visualization and Image Processing}

Immunostained sections were examined on a Zeiss Axioskop microscope equipped with appropriate filters and AxioCam digital microscope camera (Carl Zeiss Vision GmbH, Germany). Microscopy and image processing were described elsewhere [10]. Images shown are representative of at least 3 independent experiments, which gave similar results. The images were acquired at 96 DPI and submitted with the final revision of the manuscript at $300 \mathrm{DPI}$.

\section{Western Blotting Procedure}

Pancreas probes were homogenized in modified RIPA-buffer (50mM Tris-HCL pH 7.4, 150mM NaCl, 1\% NP-40, 0.25\% Na-Deoxycholate, complete protease inhibitor cocktail (Roche, Mannheim, Germany) and incubated for 20 min on ice. After 15 min centrifugation $(20000 \mathrm{~g}, 4 \mathrm{oC})$ protein concentration of supernatant was determined using BCA-kit (Pierce, Rockford, USA). For better separation protein lysates were cleaned using cleanup-kit (GE Healthcare, Amersham, Freiburg, Germany). Proteins $(50 \mu \mathrm{g})$ were separated in $6 \%$ acrylamide gels and transferred over night with 30V onto PVDF membranes (trans-blot cell apparatus, Biorad, München, Germany).

Transfer onto membrane was checked using fast green stain. After destaining, blots were blocked for $1 \mathrm{~h}$ in $5 \%$ nonfat skim milk (solved in TBS with $0.1 \%$ Tween20). First antibodies were diluted in blocking solution (NOS1 1:500, NOS3 1:250, all from Transduction Laboratories) and incubated on the blots overnight at 4oC. Positive controls were for NOS1 rat cerebrum lysate, for NOS3 human endothelial cell lysate, all from Transduction Laboratories. Signals were detected by chemiluminescence using ECLplus system (GE Healthcare, Amersham, Freiburg, Germany). Comparability of protein content in the samples used was proven by determination of GAPDH expression (house-keeping protein).

\section{Results and Discussion}




\section{Experimental Animal Model}

The rats were infused with a supramaximal concentration of the CCK analogue cerulein for up to $48 \mathrm{~h}$. As expected, the time course induction of pancreatitis resulted in an incremental increase in the pathological parameters [7]. Following criteria were used to confirm that the rats in the experimental group developed pancreatitis: macroscopic appearance of the pancreas, increase in pancreatic wet/dry-weight ratio, histological examination, transformation of acinar cells with their conversion to the morphology of ductal cells, interstitial oedema, inflammatory cell infiltration, acinar cell vacuolization and raised serum amylase activities, as described elsewhere $[4,5,9,12,13]$. Pancreas recovery was almost completed after $48 \mathrm{~h}$. Cerulein induced pancreatitis has similar pathophysiological properties compared to human edematous pancreatitis, manifesting with dysregulation of digestive enzyme production and cytoplasmic vacuolization, the death of acinar cells, edema formation, and infiltration of inflammatory cells into the pancreas $[14,15]$.

\section{NOS Expression Pattern in Pancreatitis}

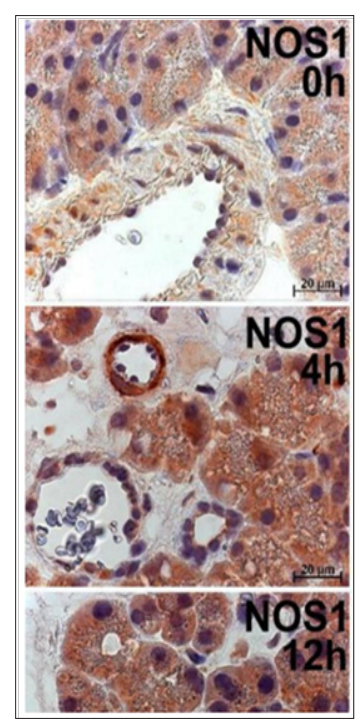

Figure 1: The same pattern of NOS expression dynamic was registered also in this study on the cerulein-induced rat pancreatitis.

Earlier, we reported that, in contrast to the commonly accepted view, all three NOS isoforms are expressed not only in pancreatic islets [16-19], but also in the exocrine compartments and in the vasculature. In pancreatitis, the expression of NOS was up-regulated especially within the exocrine compartment $[4,5]$, which reflects an exceptional vulnerability of the exocrine parenchyma. In the time course of the PDL-induced rat pancreatitis [4], dynamical changes in the expression of all three NOS isoforms, NOS1, NOS2 and NOS3, run in parallel. The same pattern of NOS expression dynamic was registered also in this study on the cerulein-induced rat pancreatitis (Figure 1). The time course induction of acute pancreatitis in the present study resulted in an incremental increase in the NOS immunostaining in the cells of exocrine pancreas with a peak at 4 and $12 \mathrm{~h}$, especially in acini and in invading inflammatory cells. As the pancreas recovery was almost completed after $48 \mathrm{~h}$, all three NOS isoforms expression returned practically to the normal level to that time.

Because of a rather high positive basal level of NOS immunostaining in Langerhans islets of normal pancreas we were unable to register any noteworthy NOS up-regulation of in the pancreatic islets in pancreatitis (not shown). In the course of pancreatitis, the ratio of cells with positively immunoreacting nuclei in the exocrine component of the pancreas drastically increased (Figure 1). Earlier, we have reported on the nuclear localization of NOS in cultured neonatal rat cardiomyocytes [20] and in muscular fibers of patients with Duchenne muscular dystrophy [21]. It seems that a similar control of NO signalling in nuclei intervened in many cell types under physiological or path physiological conditions [22]. The localization or migration of the NOS isoforms into the nucleus might provide a subcellular environment more suitable than cytosol for a more specific and effective action of NO-producing systems, and for their more direct nuclear control.

In the vasculature, vascular smooth muscle exhibited a marked expression of NOS1, whereas the intima of larger blood vessels revealed as a rule a positive immunoreaction only NOS3, like shown by us earlier for the human pancreas [23]. This is also in accord with indications in the literature that the media of blood vessels possess different physiological characteristics and differently respond to established relaxants such as acetylcholine even in the endothelium-independent mode [24]. Because of a rather high positive basal level of NOS1 immunostaining in the media of blood vessels in normal pancreas, we were unable to register any noteworthy NOS up-regulation of in the vasculature in the course of pancreatitis. The time-course of NOS expression in the ceruleininduced rat pancreatitis was confirmed by Western blotting (Figure 2) with anti-NOS1 and anti-NOS3 antibodies showing the presence of characteristic immunoreacting protein bands for NOS1 (155 $\mathrm{kDa})$ and NOS3 (140 kDa).

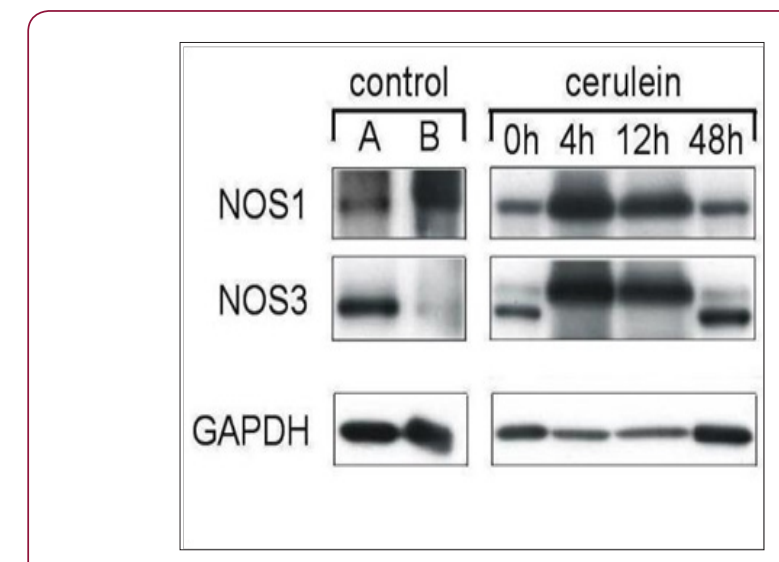

Figure 2: The time-course of NOS expression in the cerulein-induced rat pancreatitis was confirmed by Western blotting.

Western blotting of pancreatic NOS protein expression during 48h pancreatitis time-course experiments revealed marked NOS up-regulation at the time points of 4 and $12 \mathrm{~h}$, which is in agreement 
with data on NO overproduction by acinar cells found in response to cerulein hyperstimulation at these time points [25]. Interestingly, we detected double bands for NOS3 with a pronounced molecular weight shift to a higher molecular weight at the time points of 4 and 12h. A double protein band for NOS3 was also found earlier in the Western blots of blood vessels [23]. The shift in molecular weight of NOS3 during an acute pancreatitis may account for a strong phosphorylation of the protein. Phosphorylation of NOS3 at several sites is involved in the activation of nitric oxide synthesis [26].

\section{Expression Pattern of Oxidative Stress Markers-Nitroty- rosine and 80HG-in Acute Pancreatitis}

Nitrotyrosine formed by peroxynitrite-mediated nitration of protein tyrosine residues is a marker of oxidative protein damage $[27,28]$, whereas $80 \mathrm{HG}$ is a biomarker of oxidative DNA base damage [29]. In the course of cerulean-induced pancreatitis, the expression of both oxidative stress footprints - nitrotyrosine and $80 \mathrm{HG}$ - reached its maximum as early as at $30 \mathrm{~min}$, persisted till $4 \mathrm{~h}$ and markedly decreased to $12 \mathrm{~h}$ (Figure 3), whereas the peak of NOS expression was registered at 4 and $12 \mathrm{~h}$ time point. In contrast to positive immunostaining for nitrotyrosine in exocrine cells in pancreatitis, no discernible increase was detected in the vasculature, like it was earlier reported for pancreas of patients with chronic pancreatitis [5]. Pancreatic islets revealed a significant immunoreactivity to anti-nitrotyrosine antibodies also in normal pancreas (not shown), like it was also reported earlier by Nakazawa et al. [30].

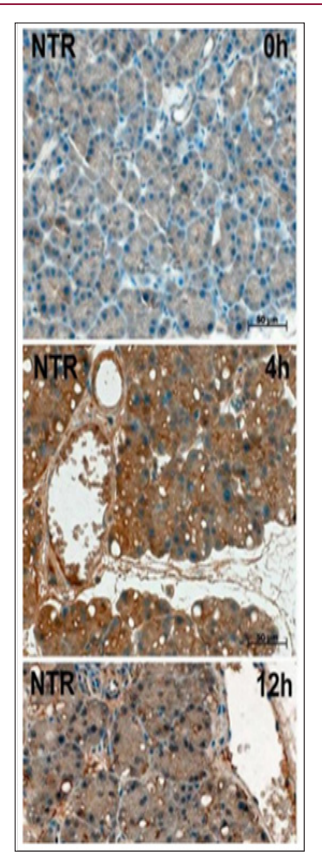

Figure 3: The expression of both oxidative stress footprints - nitrotyrosine and 8OHG - reached its maximum as early as at $30 \mathrm{~min}$, persisted till $4 \mathrm{~h}$ and markedly decreased to $12 \mathrm{~h}$.

The constitutive expression of nitrotyrosine implies that nitration might be an important physiological pathway of NO metabolism, and not merely a footprint of oxidative stress. 80HG, a biomarker of oxidative DNA base damage, is an oxidative derivative of guanosine, a modified base which occurs in DNA as a result of oxidative stress [29]. According to the manufacturer, goat antiserum to 8-hydroxyguanosine (80HG) cross reacts also with 8-hydroxydeoxyguanosine $(80 \mathrm{HdG})$; this was proved by us with preabsorption control with a 10 -fold molar excess of corresponding substrates. Figure 4 demonstrates a dramatic increase in $80 \mathrm{HG}$ immunostaining in the initiation of acute pancreatitis. 80HG immunostaining persists at this level till for $4 \mathrm{~h}$. As after $48 \mathrm{~h}$ pancreas recovery is almost completed, $80 \mathrm{HG}$ expression tends to return to the normal level. Enhanced 80HG immunostaining in pancreatitis was observed both in the cytoplasm and in the nuclei of exocrine pancreatic cells.

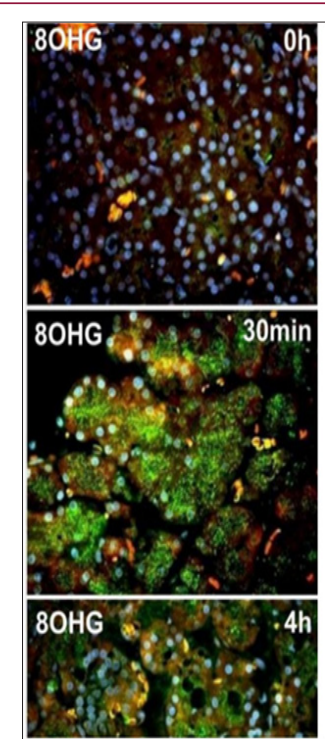

Figure 4: demonstrates a dramatic increase in $8 \mathrm{OHG}$ immunostaining in the initiation of acute pancreatitis.

Cytoplasmic immunostaining is indicative of a pronounced oxidative damage also of cytoplasmic nucleic acids. Our findings indicate that oxidative protein modifications and oxidative DNA base damages are very early events in the initial $30 \mathrm{~min}$ of cerulein-induced pancreatitis, which is in accord with the report of Reinheckel et al. [31] that protein oxidation precedes the formation of the lipid peroxidation product malondialdehyde, as well as edema and the increase in serum amylase. It implies that the outbreak of oxidative stress paves the way for the up-regulation of NOS in pancreatitis and that the NOS up-regulation is secondary in pancreatitis development being likely an adaptive mechanism aimed at maintaining the homeostatic cellular level of the bioactive NO.

\section{NO in Pancreatitis: Interplay between NO and Superox- ides}

The boundary between biology and pathobiology of the NO signaling is demarcated by the delicate balance between superoxides production rate and the ability of the body to maintain the homeostatic cellular level of the bioactive NO. In view that superoxides, known as NO scavenger, remove NO from its regular physiological course thereby reducing NO bioactivity and bioavailability, the interception of free radical reactions using antioxidants received attention by a number of investigators. Antioxidant therapy may alleviate the symptoms or prevent the 
development of pancreatitis. However, dosage levels and duration of antioxidant treatment should be carefully determined, since chronic administration of high doses of antioxidants may have deleterious effects [32]. Of equal importance is the time point of beginning of the antioxidant therapy. Some studies were able to demonstrate beneficial effects of antioxidants on parameters of pancreatic injury and acute pancreatitis like survival, edema, hyperamylasemia and macroscopic or histologic alterations, when the antioxidants where administered prior to the induction of experimental pancreatitis [33,34].

However, the attempts to treat the experimental animals with different regimens of antioxidative therapy after onset of pancreatitis were not successful [31]. Differences in the success rate of studies that administered antioxidants therapeutically during or after initiation of experimental pancreatitis may be explained by the fact that oxidative stress and oxidative protein and DNA modifications are very early events in the initial period of cerulein pancreatitis preceding NOS up-regulation. The differences may also be explained by the complex and sometimes paradoxical interplay between NO and superoxides. In inflammatory diseases like pancreatitis, superoxides up-regulate also other enzymes engaged in the L-arginine-NO-cGMP signaling, including cyclic nucleotide phosphodiesterases (PDE) [35] and arginase [36]. NO activates SGC [37], and the latter converts GTP to cGMP. PDE deteriorates NO signaling by degrading cGMP to its inactive isomer, GMP.

Arginase restricts NO generation on a competitive basis. As a result, getting short of bioactive NO the cell responses with upregulation of NOS and enzymes engaged in detoxification of ROS, including catalase and superoxide dismutase [38]. In more detail, the NO/superoxides signalling pathways in pancreatitis were discussed in our previous publication [5]. Well-defined, clinical studies involving samples from patients suffering from pancreatitis are therefore needed to validate the role of oxygen radicals in this disease. Restoration of the bioactive NO level with exogenous NO donors or supplementation with antioxidants that are deficient in patients with pancreatitis might be a feasible option for designing new adjunctive therapies for pancreatic disorders.

\section{References}

1. Tandon RK, Garg PK (2011) Oxidative stress in chronic pancreatitis: pathophysiological relevance and management. Antioxid Redox Signal 15(10): 2757-2766.

2. Andraus W, Jukemura J, Dutra F, Bechara E, Cunha JE, et al. (2007) Oxidative stress is enhanced by hypothermia imposed on ceruleininduced pancreatitis in rats. Clinics (Sao Paulo) 62(4): 483-490.

3. Mc Cord JM (2002) Superoxide dismutase in aging and disease: an overview. Methods Enzymol 349: 331-341.

4. Buchwalow I, Schnekenburger J, Atiakshin D, Samoilova V, Wolf E, et al. (2017) Oxidative stress and NO generation in the rat pancreatitis induced by pancreatic duct ligation. Acta Histochem 119(3): 252-256.

5. Buchwalow I, Schnekenburger J, Tiemann K, Samoilova V, Bankfalvi A et al. (2013) L-arginine-NO-cGMP signalling pathway in pancreatitis. Sci Rep 3: pp. 1899.

6. Chvanov M, Petersen OH, Tepikin A (2005) Free radicals and the pancreatic acinar cells: role in physiology and pathology, Philos Trans R Soc Lond B Biol Sci 360: 2273-2284.

7. Schick V, Scheiber JA, Mooren FC, Turi S, Ceyhan GO, et al. (2014) Effect of magnesium supplementation and depletion on the onset and course of acute experimental pancreatitis. Gut 63: 1469-1480.

8. Weber IA, Buchwalow I, Hahn D, Domschke W, Lerch MM, et al. (2010) The potential role of kinesin and dynein in Golgi scattering and cytoplasmic vacuole formation during acute experimental pancreatitis. Cell Res 20: 599-602.

9. Schnekenburger J, Mayerle J, Kruger B, Buchwalow I, Weiss FU, et al. (2005) Protein tyrosine phosphatase kappa and SHP-1 are involved in the regulation of cell-cell contacts at adherens junctions in the exocrine pancreas. Gut 54: 1445-1455.

10. Buchwalow IB, Boecker W (2010) Immunohistochemistry: Basics and Methods, $1^{\text {st }}$ (edn.), Springer, Heidelberg, Dordrecht, London, New York, USA.

11. Buchwalow I, Samoilova V, Boecker W, Tiemann M (2011) Non-specific binding of antibodies in immunohistochemistry: fallacies and facts. Sci Rep pp 1-28.

12. Lerch MM, Adler G (1994) Experimental animal models of acute pancreatitis. Int J Pancreatol 15: 159-170.

13. Strobel O, Dor Y, Stirman A, Trainor A, Fernandezdel Castillo C, et al. (2007) Beta cell transdifferentiation does not contribute to preneoplastic/metaplastic ductal lesions of the pancreas by genetic lineage tracing in vivo. Proc Natl Acad Sci USA 104: 4419-4424.

14. Kim H (2008) Cerulein Pancreatitis: Oxidative Stress, Inflammation, and Apoptosis. Gut and Liver 2: 74-80.

15. Hyun JJ, Lee HS (2014) Experimental Models of Pancreatitis. Clinical Endoscopy 47: 212-216.

16. Alm P, Ekstrom P, Henningsson R, Lundquist I (1999) Morphological evidence for the existence of nitric oxide and carbon monoxide pathways in the rat islets of Langerhans: an immunocytochemical and confocal microscopical study. Diabetologia 42: 978-986.

17. BurrellMA, Montuenga LM, Garcia M, Villaro AC (1996) Detection of nitric oxide synthase (NOS) in somatostatin-producing cells of human and murine stomach and pancreas. JHistochemCytochem 44: 339-346.

18. Lajoix AD, Reggio H, Chardes T, Peraldi Roux S, Tribillac F, et al. (2001) A neuronal isoform of nitric oxide synthase expressed in pancreatic betacells controls insulin secretion. Diabetes 50: 1311-1323.

19. Yu W, Niwa T, MiuraY, Horio F, Teradaira S, et al. (2002) Calmodulin over expression causes $\mathrm{Ca}(2+)$-dependent apoptosis of pancreatic beta cells, which can be prevented by inhibition of nitric oxide synthase. Lab Invest 82: $1229-1239$.

20. Buchwalow IB, Schulze W, Kostic MM, Wallukat G, Morwinski R (1997) Intracellular localization of inducible nitric oxide synthase in neonatal rat cardiomyocytes in culture. Acta Histochem 99: 231-240.

21. Buchwalow IB, Minin EA, Muller FU, Lewin G, Samoilova VE, et al. (2006) Nitric oxide synthase in muscular dystrophies: a re-evaluation. Acta Neuropathol 111: 579-588.

22. Giordano A, Tonello C, Bulbarelli A, Cozzi V, Cinti S, et al. (2002) Evidence for a functional nitric oxide synthase system in brown adipocyte nucleus. FEBS Lett 514: 135-140.

23. Buchwalow IB, Podzuweit T, Bocker W, Samoilova VE, Thomas S, et al. (2002) Vascular smooth muscle and nitric oxide synthase. FASEB J 16: 500-508.

24. Buchwalow IB, Cacanyiova S, Neumann J, Samoilova VE, Boecker W, et al. (2008) The role of arterial smooth muscle in vasorelaxation. Biochem Biophys Res Commun 377: 504-507. 
25. Ang AD, Adhikari S, Ng SW, Bhatia M (2009) Expression of nitric oxide synthase isoforms and nitric oxide production in acute pancreatitis and associated lung injury. Pancreatology 9: 150-159.

26. Fleming I, Busse R (2003) Molecular mechanisms involved in the regulation of the endothelial nitric oxide synthase, Am J Physiol Regul Integr Comp Physiol 284: R1-12.

27. Beckman JS (1996) Oxidative damage and tyrosine nitration from peroxynitrite. Chem Res Toxicol 9: 836-844.

28. Sampson JB, Rosen H, Beckman JS (1996) Peroxynitrite-dependent tyrosine nitration catalyzed by superoxide dismutase, myeloperoxidase, and horseradish peroxidase. Methods Enzymol 269: 210-218.

29. Cattleyas RC, Glover SE (1993) Elevated 8-hydroxydeoxyguanosine in hepatic DNA of rats following exposure to peroxisome proliferators: relationship to carcinogenesis and nuclear localization. Carcinogenesis 14: 2495-2499.

30. Nakazawa H, Fukuyama N, Takizawa S, Tsuji C, Yoshitake M, et al. (2000) Nitrotyrosine formation and its role in various pathological conditions. Free Radic Res 33: 771-784.

31. Reinecker T, Priuses J, Nedelev B, Augustin W, Schulz HU, et al. (1999) Oxidative stress affects pancreatic proteins during the early pathogenesis of rat caerulein pancreatitis. Digestion 60: 56-62.

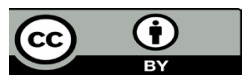

This work is licensed under Creative Commons Attribution 4.0 License

Submission Link: https://biomedres.us/submit-manuscript.php
32. Yu JH, Kim H (2014) Oxidative stress and inflammatory signaling in cerulein pancreatitis. World J Gastroenterol 20: 17324-17329.

33. Munoz Casares FC, Padillo FJ, BricenoJ, Collado JA, Munoz Castaneda JR, et al. (2006) Melatonin reduces apoptosis and necrosis induced by ischemia/reperfusion injury of the pancreas. J Pineal Res 40: 195-203.

34. Esrefoglu M (2012) Experimental and clinical evidence of antioxidant therapy in acute pancreatitis. World J Gastroenterol 18: 5533-5541.

35. Gomez Cambronero L, Camps B, de La Asuncion JG, Cerda M, Pellin A, et al. (2000) Pentoxifylline ameliorates cerulein-induced pancreatitis in rats: role of glutathione and nitric oxide. J Pharmacol Exp Ther 293: 670-676.

36. Waddington SN (2002) Arginase in glomerulonephritis. Kidney Int 61: 876-881.

37. Martin E, Berka V, Tsai AL, Murad F (2005) Soluble guanylyl cyclase: the nitric oxide receptor. Methods Enzymol 396: 478-492.

38. Mc Cord JM (2002) Superoxide dismutase in aging and disease: an overview. Methods Enzymol 349: 331-341.

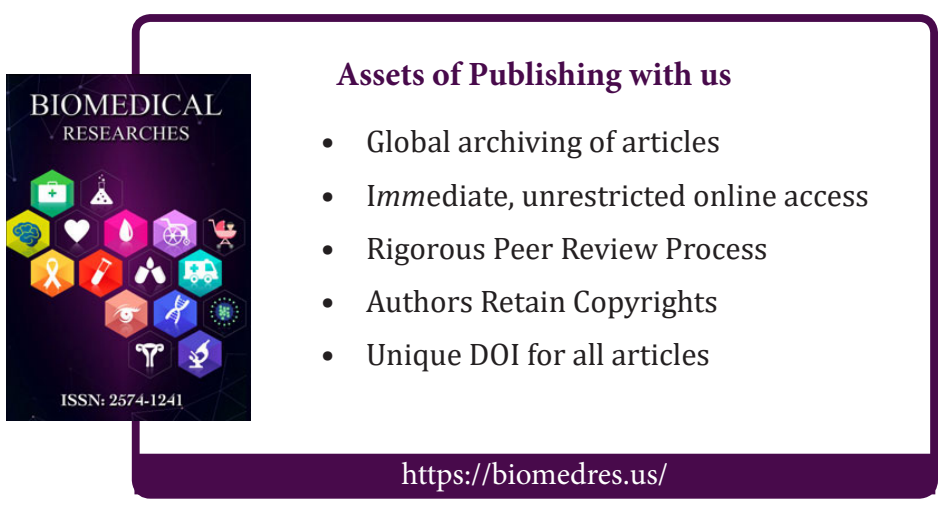

